\title{
EMC ISSUES FOR WIND TURBINES
}

\author{
M.C. Esteban ${ }^{1}$, F. Arteche $^{1}$, M. Iglesias ${ }^{1}$ and FJ. Arcega ${ }^{2}$ \\ ${ }^{1}$ Instituto Tecnológico de Aragón (ITA) \\ María de Luna 7-8, 50018 Zaragoza (Spain) \\ Phone:+34 976010042 , e-mail: cesteban@ita.es \\ ${ }^{2}$ Department of Electrical Engineering \\ University of Zaragoza \\ María de Luna 3, 50018 Zaragoza (Spain) \\ Phone:+34 976 762152, e-mail: arcegafi@unizar.es
}

\begin{abstract}
This paper addresses the study of the immunity to common mode spurious signals of any generic electronic system used in wind turbines. This study uses numerical simulations based on a MTL model applied to shielded cables to analyze the effect of common mode signals in the control system of wind turbines and the impact on the common mode immunity of different design parameters on the front-end. The generic set-up is composed by the transducer and input circuitry of the control located about 10 meters from the transducer. Shielded cables are used to connect the transducer to the input amplifiers. This study allows predicting electromagnetic compatibility (EMC) problems and estimating the effect of interference noise at early stages of the design.
\end{abstract}

\section{Key words}

Wind power, electromagnetic compatibility, EMI, Multi conductor transmission line, electronics integration, renewable energies, functional safety.

\section{Introduction}

Wind power energy is one of the most important renewable energies [1]. During the last years, the high demand of renewable energies has increased the number and the size of the wind turbines. For that purpose manufacturers have recently turned to more complex turbines to capture power over a wide range of wind speeds and to increase the power of the turbines. These turbines require more electronic systems [2] that need the careful selection of the input topology and design to avoid that electromagnetic interferences (EMI) affect the satisfactory system operation.

Last generation of wind turbines requires complex systems to operate. As turbines become larger and more flexible, advanced electronics becomes necessary to meet multiple objectives. In general, the electronic systems can be used to monitor the condition of the wind turbine, measure the rotational speed of the rotor and generator voltages and currents. On recent wind turbine models, some sensitive components of these systems are usually connected using fibre optics, however some of them are still connected via copper cables which become very susceptible to EMI phenomena. This electromagnetic compatibility (EMC) scenario introduces a large amount of problems during the design and integration of the electronics systems. It requires new methods and careful design to ensure the correct integration of the electronic systems to ensure the reliable operation and good performance of the wind turbine.

The purpose of this paper is to present the assessment of EMC in the design of the electronic systems for wind turbines. It addresses the studies and methods that have been carried out to guarantee the correct integration of the electronics to meet the new growing challenges of the wind turbines.

\section{Sensitive electronics}

The electromagnetic environment of wind turbines is becoming more complex because of the increase of power and the amount of electronic systems installed. High level noise sources and very sensitive equipment are needed to work together in a very small area increasing the possibility of failure due to electromagnetic interference. The control system is one of the most sensitive circuits of wind turbines. Wind turbines have recently started to increase the power installed in each unit, which is forcing the development of a bigger nacelle that requires longer cables to connect different units of the system (control and power units,). As a result, the sensitive electronic systems of the wind turbine have to be placed in remote areas from the transducers. This topology sets different ground potentials (high frequency) between the transducers and the control front-end circuitry. Fig. 1 shows a simple 
schematics of a wind turbine's control system when the transducer is connected to the front-end via a cable.

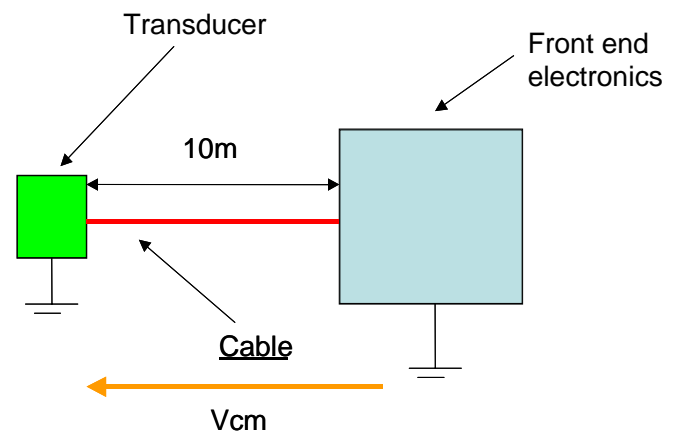

Fig. 1.-Topology under study

This structure is very sensitive to EMI phenomena and any electromagnetic noise must be kept very low in order to fulfil the performance requirements and safe operation. The different components and grounding topologies of this circuit have been modelled by a set of impedances that has been changed during the analysis to evaluate the best configuration of the input connection. This paper addresses the study of the immunity to common mode spurious signals perturbing the control electronic system described above. It allows predicting grounding and shielding problems and estimating the effect of interference noise at early stages of the design.

The common mode rejection of the topology depicted in Fig. 1 is analysed and the effects of different impedances and connections of the traducer and input control system are addressed. The influence of these parameters has been studied by simulation using a model of the cables based on the multi-transmission line theory (MTL) [3][4]. These quantitative studies are important to design systems with high immunity and address the susceptibility to interference noise during the design stage. This analysis is part of the EMC-based design approach to define the type of cable (shielded or unshielded), shield connections, filters and configuration of the traducer / front-end electronics required to ensure the good performance of the wind turbine and the functional safety of the system.

\section{Multi-conductor transmission line model}

The studies are conducted using a model for the cable based on the multi-conductor transmission line (MTL) theory [3][4]. This model assumes transverse electromagnetic (TEM) wave as the propagation mode. As such, the components of both the electric and magnetic fields lie in a plane transverse to the direction of propagation satisfying a static distribution in the perpendicular plane. It allows representing the cable model in per-unit parameters that are relatively easy to measure or calculate given the material properties and geometry of the cable.

Due to the skin effect in the shield, currents and voltage on the shield can be separated in inner and outer. This behaviour on the shield allows separating the model of the complete multi-conductor cables with shield in two parts. The inner system consists of the inner conductor of the cable and the inner face of the shield as reference. The outer system models the interaction of the environment with the shield and includes the effect of propagation of signals between the shield and the structure of the system.

\section{A. Inner conductors - Model}

The inner conductors of a multi-conductor transmission line can be modelled representing the cable by $\mathrm{N}$ conductors and taking as reference conductor for the line voltages the inner face of the shield. The transmission line is considered uniform such that the $\mathrm{N}$ conductors are parallel to each other and with respect to the shield and the dielectric properties are independent of the geometric coordinates. Setting the cable in a rectangular coordinate system, the direction of propagation is coincident with the $\mathrm{z}$ axis of that frame. The per-unit-length equivalent circuit of the MTL with shield of infinitesimally length $\Delta \mathrm{l}$ is represented in Fig. 2. The inner conductor model corresponds to the upper part of the scheme.

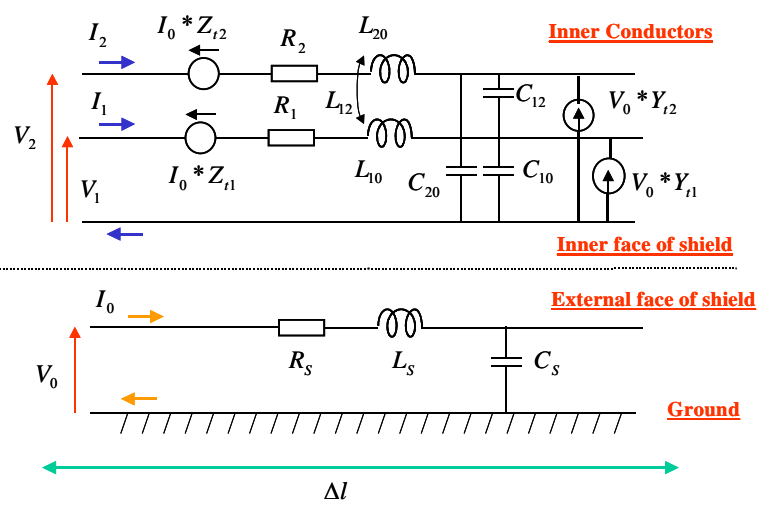

Fig. 2. Per-unit-length equivalent circuit for a shielded multi-conductor transmission line.

Assuming a MTL sector with infinitesimal length, $\Delta \mathrm{l}$, as it is depicted in Fig. 2, the inner conductors can be modelled by the partial differential equation:

$$
\begin{aligned}
& \frac{\partial}{\partial z} V(z, t)=-R I(z, t)-L \frac{\partial}{\partial t} I(z, t) \\
& \frac{\partial}{\partial z} I(z, t)=-G V(z, t)-C \frac{\partial}{\partial t} V(z, t)
\end{aligned}
$$

where $I(z, t)$ and $V(z, t)$ are vectors representing the current and the voltage, respectively, in each conductor respect to the reference conductor; $L, C, R, G$, are $\mathrm{NxN}$ matrices representing the per-unit-length inductance, capacitance, resistance, and conductance of the line, respectively, $z$ is the position along the transmission line and $t$ denotes the time variable.

\section{B. Shield model: Surface Transfer Impedance}

The complete model of the cable including the shield consists of the inner system representing the central conductors and the inner part of the shield augmented by the coupling effect of voltage and currents flowing through the shield [5][6]. 
The outer system (shield - environment) is considered as a transmission line, where the metallic tray is the reference of the outer system and the shield is the conductor. The voltage and current sources at each inner conductor represent the interaction between both inner and outer systems. $Z t$ and $Y t$ represent the surface transfer impedance and the surface transfer admittance. The transfer impedance $(\mathrm{Zt})$ is defined as the ratio between the voltage of the inner conductor i respect to the shield and the current flowing through the shield, per unit length. The transfer admittance is defined as the ratio between the current flowing through the inner conductor and the voltage between the shield and the environment, per unit length. The last magnitude is generally very small and it has not been considered in the present study. Both, the surface transfer impedance and admittance are characteristic parameters of the shield of the cable.

The transfer impedance depends on three components as defined by the equation [6][7][8].

$$
Z t=Z d(\omega)+j \cdot \omega \cdot(M h \pm M b)
$$

where: $Z d(\omega)$ : The Diffusion coupling component is due to skin effect in the shield. It is predominant at low frequencies.

Mh : The aperture-coupling component is defined as the coupling through the holes of the shield. It plays an important role in the value of the transfer impedance at high frequencies.

$M b$ : The braid inductance component is defined as the coupling between the external and the internal layers of the shield.

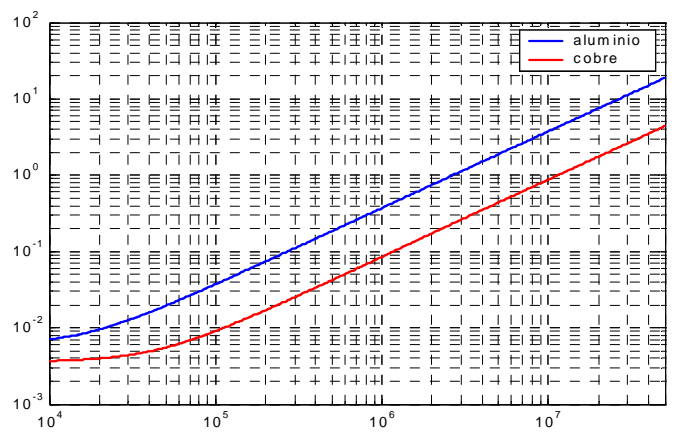

Fig. 3: Surface transfer impedance of copper and aluminium shield

To include the effect of the surface transfer impedance and admittance in the inner conductors, the mathematical model defined by Eqn. 1 is augmented by the generators Zt.Io(z,t) and Yt.Uo(z,t).

$$
\begin{aligned}
& \frac{\partial}{\partial z} V(z, t)=-R I(z, t)-L \frac{\partial}{\partial t} I(z, t)+Z t . I o(z, t) \\
& \frac{\partial}{\partial z} I(z, t)=-G V(z, t)-C \frac{\partial}{\partial t} V(z, t)+Y t \cdot U o(z, t)
\end{aligned}
$$

The solution of the complete system of equations starts with the solution of the outer system, calculating the distributed voltage $\operatorname{Uo}(\mathrm{z}, \mathrm{t})$ and the distributed current $I o(z, t)$ at every location $z$ of the outer system. These voltages and currents are used to calculate the magnitude of the additional generators defined in (3). After that, Eqn. 3 is solved calculating the general solution of the MTL equation in frequency-domain and incorporating the terminal network constraints in the general solution to determine the line voltages and currents at the both ends of the line.

\section{Control systems for wind turbines}

The sensing circuitry of the wind turbine's control system is composed by three main elements.

- Transducer,

- Control electronics (Input Amplifier)

- Cable.

For large wind turbines, this circuit is characterised by the long distance between the transducer and front-end control electronics. This structure is very sensitive to common mode spurious signals and the electromagnetic noise must be kept low in order to fulfil the performance requirements. The common mode immunity of the system topology has been studied considering the circuit depicted in figure 4.

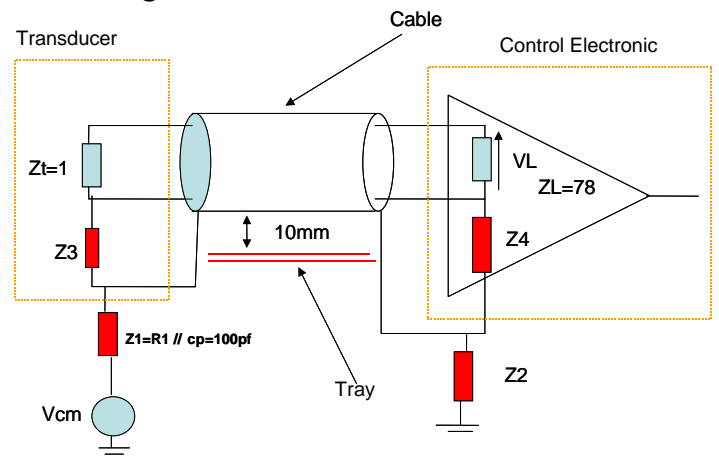

Fig. 4. Equivalent circuit to analyze the common mode rejection of the system.

The different components and grounding topologies of this circuit have been modelled by a set of impedances that have been changed during the analysis. The control electronics has been modelled by its input stage. This stage is characterized by the input impedance of the amplifier $\left(Z_{L}\right)$, whose value is equal to the characteristic impedance of the cable. The impedance $Z_{4}$ represents the common-mode input impedance of the amplifier, which can change between $Z_{4}=0$ ohms (single-end topology) and $Z_{4} \rightarrow \infty$ ohms (ideal differential amplifier). There is a wide range of transducers sensing different variables of the turbine, e.g. voltages, currents or rotor positions/velocities. These devices may be modelled by the output impedance $(\mathrm{Zt})$ which has a value between 0.1 to $1 \mathrm{ohm}$. For the present study a shielded twisted pair cable TWC-78 placed $10 \mathrm{~mm}$ above a reference plane, has been chosen to connect the transducer and the control electronics located 10 meters away. It is a common cable and there is plenty of information and tests about this cable that allowed a simple validation of the model used in this study. The magnitude of the inductance and capacitance matrix for the internal and external circuit has been calculated based on measurements and numerical methods. 


\section{A. External system impedances: shielding-metallic tray circuit}

The external system has represented by a circuit made by the shielding and the metallic tray. This circuit could be study by a transmission line were the metallic try is the reference point of the external system and the shielding is the conductor. The parameters for this transmission line has been calculated by the inductance and capacitance of a cable which radio is $1,65 \mathrm{~mm}$ and is $10 \mathrm{~mm}$ between the cable and the reference plane [3 ]

$$
\begin{aligned}
L_{e x t} & =[0.496] \times 10^{-6} \frac{\mathrm{H}}{\mathrm{m}} \\
C_{\text {ext }} & =[22,4] \times 10^{-12} \frac{\mathrm{F}}{\mathrm{m}}
\end{aligned}
$$

The shielding resistance has been measured and based on these measurements a model [3], has been created to include the skin effect.

$$
R_{e x t}=\left[0,014 \cdot\left(1+\sqrt{\frac{\omega}{250 \times 10^{3}}}\right)\right] \frac{\Omega}{m}
$$

\section{B. Internal impedance: cables-Shield circuit}

The internal circuit is defined by the central conductors and the shield, which is the reference for the transmission line. The inductances, capacitances and resistances have been measured and the matrix magnitudes are:

$$
\begin{gathered}
L_{\mathrm{int}}=\left[\begin{array}{cc}
0,219 & 0,0225 \\
0,0225 & 0,230
\end{array}\right] \times 10^{-6} \frac{H}{m} \\
C_{\mathrm{int}}=\left[\begin{array}{cc}
98,1 & -36,6 \\
-36,6 & 95,1
\end{array}\right] \times 10^{-12} \frac{f}{m} \\
R_{\mathrm{int}}=\left[\left(0,293 \cdot\left(1+\sqrt{\frac{w}{1.47 \times 10^{6}}}\right)\right)\right. \\
\left.0 \quad\left(0,298 \cdot\left(1+\sqrt{\frac{w}{1.47 \times 10^{6}}}\right)\right)\right] \Omega
\end{gathered}
$$

\section{Common mode rejection}

The common mode immunity of the system has been evaluated by the common mode transfer function. This function is the ratio between the voltage at the input of the amplifier and the common mode voltage:

$$
T_{M C}=20 \times \log \left(\frac{V_{L}}{V_{M C}}\right)
$$

The effects of the ground connections are studied considering different values of impedances between the transducer to ground and the input amplifier to ground. In addition, the topology and quality of the amplifier and transducer are studied. For those cases, the internal impedances $Z_{3}$ and $Z_{4}$ connected to ground are changed and used as parameter to quantify their effect on the common mode rejection of the system. Finally, types of cable's shield as well as the shield connections are studied.

\section{A. Ground connexion effects of transducer and amplifier. $\left(Z_{1}-Z_{2}\right)$}

Figure 5 depicts the common mode transfer function, for a frequency range between $10 \mathrm{kHz}$ and $40 \mathrm{MHz}$, for different values of $Z_{1}(0 \Omega, 100 \Omega, 10 \mathrm{k} \Omega, y 1 \mathrm{M} \Omega$,) with the input amplifier (control system) connected to ground $\left(Z_{2}=0\right)$. The ground impedance of the transducer has a big influence in the common mode rejection of the system at low frequencies. However there is no significant difference between the different configurations for frequencies higher than 3-4 MHz due to the parallel stray capacitance $C_{1}$ associated with this connection and the resonance of the cable.

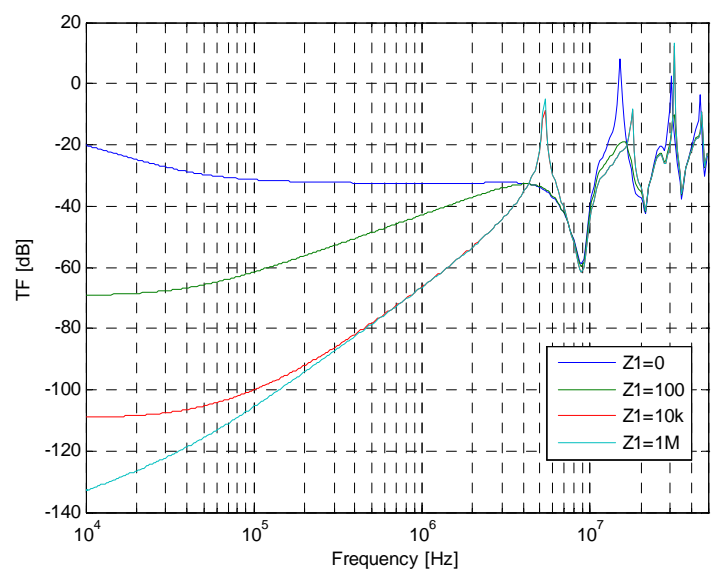

Fig. 5.- Common mode rejection: Transducer connection $\left(Z_{1}\right)$

A similar result is showed for the ground connection of the input amplifier. Figure 6 depicts the common mode transfer function, for a frequency range between $10 \mathrm{kHz}$ and $40 \mathrm{MHz}$, for different values of $\mathrm{Z} 2(0 \Omega, 100 \Omega$, $10 \mathrm{k} \Omega$, and $1 \mathrm{M} \Omega$,) with the transducer connected to ground $\left(\mathrm{Z}_{1}=0\right)$.

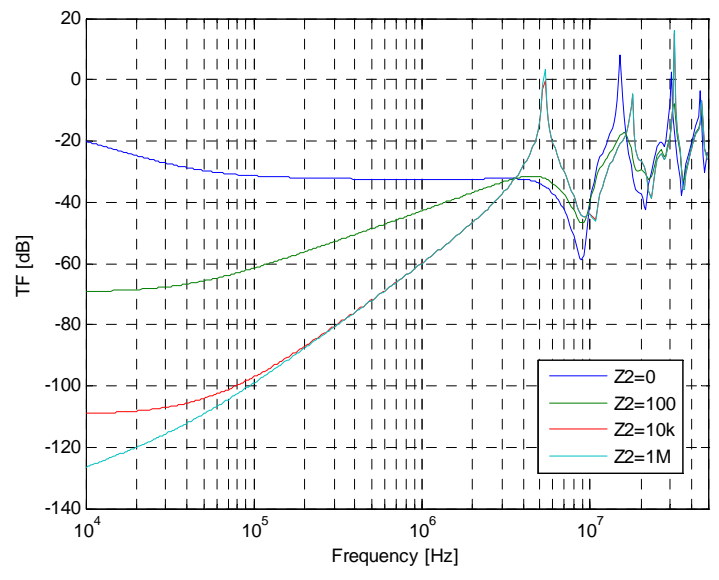

Fig. 6.- Common mode rejection: Amplifier connection $\left(\mathrm{Z}_{2}\right)$

B. Control system selection: CM rejection of the amplifier $\left(Z_{4}\right)$

This section study the common mode rejection defined by common mode input impedance of the amplifier. This amplifier may be selected with high, medium or low 
common mode input impedance. Figure 7 depicts the common mode rejection, for a frequency range between $10 \mathrm{kHz}$ and $40 \mathrm{MHz}$, for different values of $Z_{4}(0 \Omega$, $100 \Omega, 10 \mathrm{k} \Omega$, and $1 \mathrm{M} \Omega$,) with the input amplifier connected to ground $\left(Z_{2}=0\right)$. The case $Z_{4}=0 \Omega$ corresponds to the single-end connection of the input amplifier. The common mode input impedance of the amplifier has a big influence in the common mode rejection of the system. This difference is higher at low frequencies, however at high frequency this effect is lower because of cable impedances. The final selection of the amplifier has to take into account not only the performance of the system but the cost of the amplifier as the cost of the amplifier is correlated with its ability to reject the common mode signals.

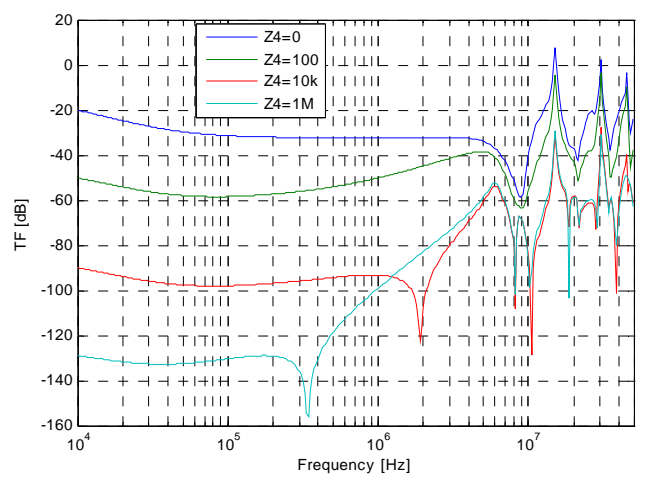

Fig. 7.- Common mode rejection: Input amplifier $\left(\mathrm{Z}_{4}\right)$

\section{Control system selection: Transducer impedance $\left(Z_{3}\right)$}

Figure 8 depicts the common mode transfer function for a frequency range between $10 \mathrm{kHz}$ and $40 \mathrm{MHz}$, for different values of $Z_{3}(0 \Omega, 100 \Omega, 10 \mathrm{k} \Omega$, and $1 \mathrm{M} \Omega$, with the transducer connected to ground $\left(Z_{1}=0\right)$. The internal impedance of the transducer has a big influence in the common mode rejection of the system at low frequencies. However there is no significant difference between the different configurations at high frequency because of cable and shield impedances.

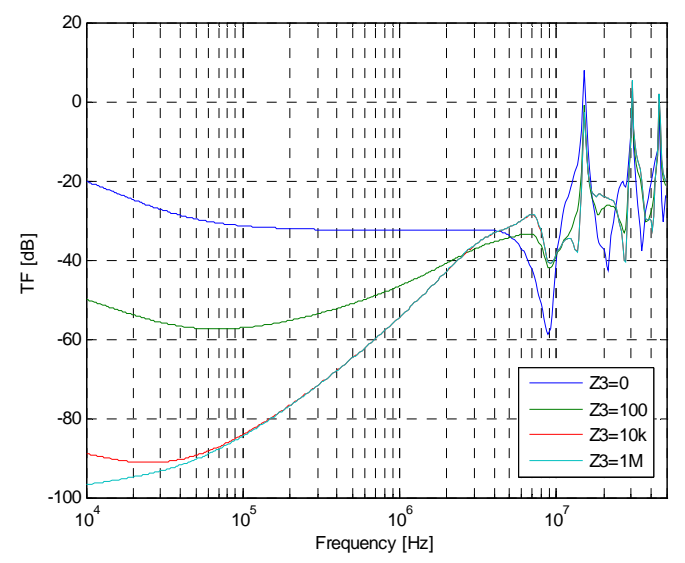

Fig. 8.- Transference function for different $\mathrm{Z} 3$

\section{Signal transmission mode: Differential \& Single end transmission}

This section presents the effects of noise currents in the two possible modes of signal transmission (Differential and single end transmission). The single-end topology has been simplified connecting the internal impedances of the transducer and amplifier directly to ground $\left(Z_{3}=0\right.$ and $Z_{4}=0$ ), whereas the differential mode transmission has been modelled increasing the value of these impedances up to $1 \mathrm{M} \Omega$,). As expected, the results depicted in figure 9 shows that the differential signal transmission is has better common mode rejection than the single end transmission.

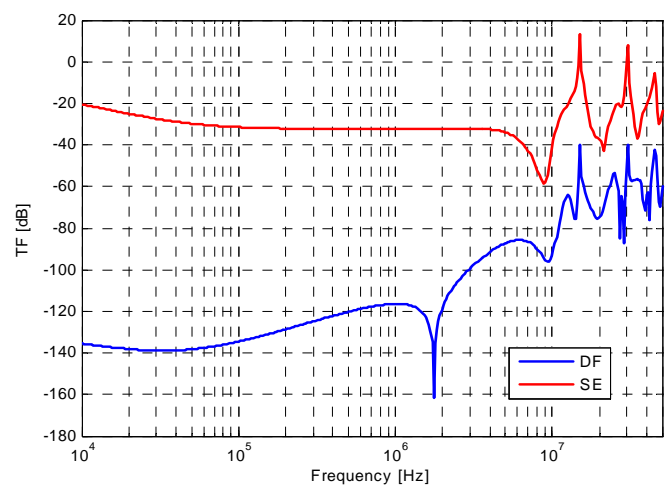

Fig. 9.- Common mode rejection: Signal transmission

\section{E. Shield properties and shield connection effects.}

The near (electric and magnetic) and far electromagnetic fields as well as high frequency ground currents induce currents in the cable shield and cable trails. As it has been explained previously, at high frequency these shield currents always flow through the external face of the shield, due to skin effect, producing some effect in the inner conductors. HF shield currents mainly generate a voltage ( $\mathrm{CM}$ voltage) in the central conductors. The value of this voltage is defined by the type of shield (material, coverage, holes etc). Fig. 10 shows the common mode transfer function considering two cables with different shields. The most important point of this plot is the large difference in screening between both shields. The aluminium shield has larger surface transfer impedance than the cooper braid and the CM rejection ratio magnitude is also large, providing lower noise immunity in the system.

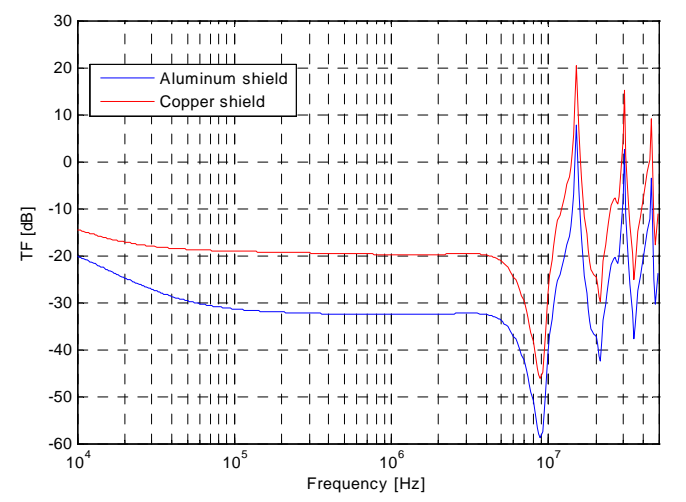

Fig. 10.- Common mode rejection : With and without shield

In the case that the shield is not present or it is connected only at one end, CM currents are induced directly in the central conductors of the power cables because the low impedance of the circuit associated with those cables. As a result the immunity of the system strongly decreases. 
Figure 11 shows the noise immunity to common mode currents of the topology under study for a cable with (in blue) and without shield (in red).

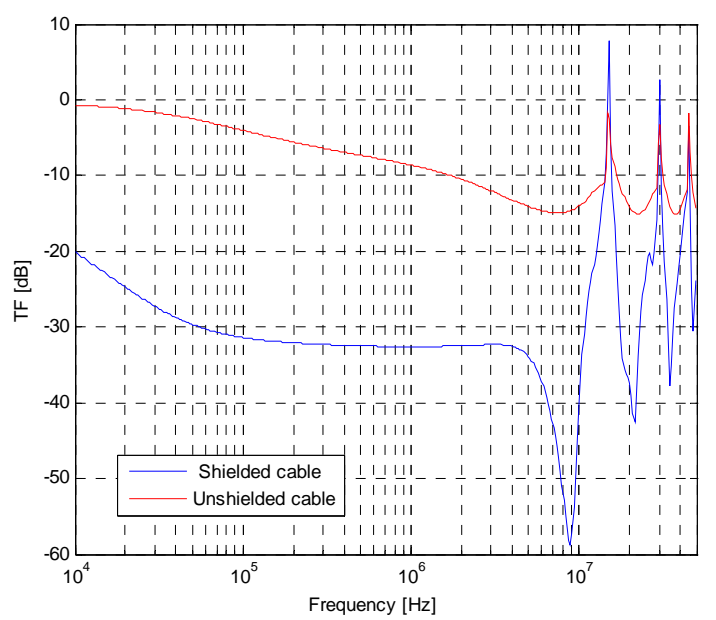

Fig. 11.- Common mode rejection : With and without shield.

The quality and connection of the shield is very important to define the amount of noise that can be coupled to the central conductors. As a consequence the final selection of the shield will depend on the susceptibility of the electronics to these CM currents. In case the control systems is very sensitive to noise, it will be necessary to use a very good shielding to improve the noise susceptibility of the system

\section{Conclusion}

This paper has presented a preliminary noise immunity analysis of the differential amplifier to be used by wind turbines. The study of the sensitivity of the control electronics to common mode currents has been conducted by simulation using a line model based on the MTL theory. Results have shown the limitations of the topology to common mode rejection if it is implemented with elements with poor common mode rejection and unshielded cables. It was shown that the type of shield and signal transmission has also an important impact. The rejection to common mode currents can be improved by selecting a topology based on differential mode transmission with twisted pair shielded cable and with components with high common mode rejection ratio. Cables impedances as well as stray component associated to ground connections decrease the immunity of the topology. .

\section{Acknowledgement}

The authors would like to thank to Instituto Tecnológico de Aragón (ITA), specially Dr. J.L. Pelegay, head of G.I.A. (Grupo de Investigación Aplicada) for the support of this work. Also they want to thank to C. Rivetta (SLAC, Stanford University USA) for the fruitful discussions.

\section{References}

[1] J.M. Carrasco and al, "Power-electronic systems for the grid integration of renewable energy sources: a survey." IEEE Trans. on Industrial electronics, vol 35, $\mathrm{n}^{\circ}$ 4, agosto 2006.

[2] F. Blaabjerg, F. Iov, R. Teodorescu, Z. Chen, “ Power electronics in renewable energy systems." IEEE Trans. on power electronics, 2006.

[3] Paul, Clayton R. "Analysis of multi-conductor transmission lines", 1992, ISBN-0-471-02080-X

[4] Arteche, F; Rivetta, C. "Noise susceptibility analysis of the HF front-end electronics for the CMS high-energy experiment" IEEE International Symposium on EMC. Volume 2, 18-22 Aug. 2003 Page(s):718 - 723 vol.2

[5] PAUL, CLAYTON R. "Introduction to Electromagnetic Compatibility" , New York, NY Wiley $1992765 \mathrm{p}$

[6] Edward F. Vance, "Coupling to shielded cables". 1987, ISBN 0-89874-949-2.

[7] Tyni M., "The transfer impedance of coaxial with braided outer conductor". Pr. Nauk, Inst Telekomun Akust. Politech Wroclaw Ser. Kon_,pp 410-419, 1975.

[8] S. Sali, "An improve Model for the Transfer Impedance Calculations of Braided Coaxial Cables", IEEE Trans. on Electromagnetic Compatibility, vol. 33, Issue 2, pp 139-143, May 1991. 